\title{
CHARACTERIZING $k$-DIMENSIONAL UNIVERSAL MENGER COMPACTA
}

\author{
BY MLADEN BESTVINA
}

The disjoint $k$-cells property $\left(D D^{k} P\right)$, isolated by J. W. Cannon $[\mathbf{C a}]$, has played the critical role in the characterization theorems for finite-dimensional manifolds (R. D. Edwards [Ed], F. Quinn [Qu]) and for manifolds modeled on the Hilbert cube (H. Torunczyk [To]). A metric space $X$ has $D D^{k} P$ if each pair $f, g: I^{k} \rightarrow X$ of maps of a $k$-cell into $X$ can be arbitrarily closely approximated by maps with disjoint images.

By Toruńczyk's characterization theorem, a compact AR is homeomorphic to the Hilbert cube $Q$ iff it satisfies $D D^{k} P$ for $k=0,1,2, \ldots$.

On the other hand, the Cantor set $C=\mu^{0}$ is the only 0-dimensional compactum that satisfies $D D^{0} P$ (i.e. does not have isolated points). From R. D. Anderson's characterization of the universal curve $\mu^{\mathbf{1}}\left[\mathbf{A n}_{\mathbf{2}}\right]$ (the 1dimensional Peano continuum with no local cut points which does not contain a nonempty open set that can be embedded into the plane), it follows that $\mu^{1}$ is the only connected $\left(C^{0}\right)$, locally connected $\left(L C^{0}\right)$ 1-dimensional compactum that satisfies $D D^{1} P$. The construction of the universal curve generalizes to give the $k$-dimensional universal Menger space $\mu^{k}$ : Subdivide $[0,1]^{2 k+1}=A_{1}$ into $3^{2 k+1}$ congruent $(2 k+1)$-cubes, and let $A_{2}$ be the union of the cubes adjacent to the $k$-skeleton of $[0,1]^{2 k+1}$. Repeat the construction on each of the remaining cubes to obtain $A_{3}$ and, similarly, $A_{4}, A_{5}, \ldots$. Then set $\mu^{k}=\bigcap_{i=1}^{\infty} A_{i}$.

THEOREM. If $X$ is a $k$-dimensional $(k-1)$-connected $\left(C^{k-1}\right)$, locally $(k-1)$-connected $\left(L C^{k-1}\right)$ compact metric space that satisfies $D D^{k} P$, then $X \approx \mu^{k}$.

COROLLARY. Different constructions of the universal $k$-dimensional space appearing in the literature ( $f f .[\mathbf{M g}, \mathbf{L f}, \mathbf{P a}])$ yield the same space.

In the proof, a different construction of $\mu^{k}$ is used as a working definition. This construction is more suitable for inductive arguments, since it allows "handlebody decompositions" of $\mu^{k}$, where each "handle" is a copy of $\mu^{k}$, the intersection of two "handles" is a copy of $\mu^{k-1}$, the intersection of three "handles" is a copy of $\mu^{k-2}$, etc. This approach leads to a construction of many homeomorphisms $h: \mu^{k} \rightarrow \mu^{k}$ which are used to develop a decomposition theory for $\mu^{k}$ (via Bing's Shrinking Criterion). The main result here is that a $U V^{k-1}$-surjection $f: \mu^{k} \rightarrow X$ is approximable by homeomorphisms provided $\operatorname{dim} X=k$ and $X$ satisfies $D D^{k} P$.

The final part of the proof consists of showing that any $C^{k-1}, L C^{k-1}$ metric compactum admits a $U V^{k-1}$-surjection $f: \mu^{k} \rightarrow X$ (a resolving map).

Received by the editors May 1, 1984 and, in revised form, May 21, 1984.

1980 Mathematics Subject Classification. Primary 54F20, 57Q99; Secondary 54F45. 
Omitting compactness and global connectivity, we obtain the characterization of manifolds modeled on $\mu^{k}$.

THEOREM. For a locally compact, locally $(k-1)$-connected $k$-dimensional metric space $X$, the following statements are equivalent.

(i) $X$ satisfies $D D^{k} P$.

(ii) Each point $x \in X$ admits a neighborhood $U$ homeomorphic to an open subset of $\mu^{k}$.

Many theorems from $Q$-manifold theory translate immediately into $\mu^{k}$ manifold theory. For example, the $Z$-set unknotting theorem (properly interpreted) holds for $\mu^{k}$-manifolds. In particular, $\mu^{k}$ is homogeneous (this fact is well known for $k=0$, and it was proved by $\mathrm{R}$. D. Anderson $\left[\mathbf{A n}_{\mathbf{1}}\right]$ for $k=1$ ). In short, $\mu^{k}$ is "the $k$-dimensional analogue of the Hilbert cube $Q$ ".

\section{BIBLIOGRAPHY}

[An 1 R. D. Anderson, A characterization of the universal curve and a proof of its homogeneity, Ann. of Math. (2) 67 (1958), 313-324.

$\left[\mathbf{A n}_{2}\right] \ldots$, One-dimensional continuous curves and a homogeneity theorem, Ann. of Math. (2) 68 (1958), 1-16.

[Ca] J. W. Cannon, Shrinking cell-like decompositions of manifolds. Codimension three, Ann. of Math. (2) 110 (1979), 83-112.

[Ed] R. D. Edwards, The topology of manifolds and cell-like maps, Proc. Internat. Congress Math. (Helsinki, 1978), Acad. Sci. Fennica, 1980, pp. 111-127.

[Lf] S. Lefschetz, On compact spaces, Ann. of Math. (2) 32 (1931), 521-538.

[Mg] K. Menger, Kurventheorie, Teubner, Leipzig, 1932.

[Pa] B. A. Pasynkov, Partial topological products, Trans. Moscow Math. Soc. 13 (1965), 153-271.

[Qu] F. Quinn, Resolutions of homology manifolds, and the topological characterization of manifolds, Invent. Math. 72 (1983), 267-284.

[To] H. Toruńczyk, On CE-images of the Hilbert cube and characterization of $Q$-manifolds, Fund. Math. 106 (1980), 31-40.

Department of Mathematics, University of Tennessee, Knoxville, TENNESSEE 37996 